\title{
Streaming vs. fusion of sinusoidal components of complex tones
}

\author{
GARY L. DANNENBRING \\ St. Francix Xavier University, Antigonish, Nova Scotia B2G 1C0, Canada
}

and

\author{
ALBERT S. BREGMAN \\ McGill University, Montreal, Quebec, Canada
}

\begin{abstract}
The present experiments looked at the effect of a number of variables on the tendency of an element of a complex tone to either fuse with the other elements of the tone or be "pulled out" into a "horizontal," temporal stream. It was found that such factors as the intensity relationship of the elements of the complex tone, the frequency of the tone being pulled out, and various factors producing temporal asynchronies between elements of the complex, all affect the tendency for either fusion or streaming to occur.
\end{abstract}

Over the last few years, a number of papers have been published dealing with auditory stream segregation of repeating sequences of sounds (Bregman \& Campbell, 1971; Bregman \& Dannenbring, 1973, in press; Bregman \& Rudnicky, 1975; Dannenbring \& Bregman, 1976a, 1976b; Bregman, 1977). Basically, this is a phenomenon in which a rapid sequence of sounds is perceived as two or more parallel streams rather than a single sequence. Although this phenomenon is illusory in nature, since there is physically one stream of sounds, the mechanism producing this effect probably evolved as a means of allowing the human organism to accurately perceive sound sequences that occur in the real world, when there are a number of cooccurring streams of sounds (Bregman \& Dannenbring, in press).

One of the limitations of most of the research to date is that it has dealt only with sequences of nonoverlapping sounds. In the real world, sounds occur both sequentially and simultaneously. The question of interest here concerns how the auditory system separates the components of a complex incoming waveform that are due to different sources, under normal conditions. This has been described by Bregman and Dannenbring (in press) as the problem faced by a person listening to an orchestra through a single loudspeaker; how is he able to hear individual

This research was supported by grants from the National Research Council of Canada and the St. Francis Xavier University Council for Research, awarded to the first author, and by grants from the National Research Council of Canada, the F.C.A.C. program of the Quebec Ministry of Education, and the McGill University Graduate Faculty, awarded to the second author. Reprints may be obtained from Dr. Gary L. Dannenbring, Department of Psychology, St. Francis Xavier University, Antigonish, Nova Scotia, B2G 1C0, Canada. instruments, which involves grouping across time, as an auditory stream, a subset of the partials occurring at that time?

A survey of the literature reveals very little research on this subject. Various studies have shown that one can, indeed, "hear out" the partials of a complex tone (Plomp, 1964; van Noorden, 1975). The question here, however, is the factors which cause groups of these partials to fuse into one complex tone, others to fuse into another tone, etc., with patterns of these tones then forming separate auditory streams. One of the few people to address himself to this problem was Helmholtz (1885). He felt that there were various sources of information which could allow the auditory system to solve this problem. An important one is that even if sounded simultaneously, different instruments produce tones with different rise times, so that the waveform envelope could be a source of information. Unfortunately, Helmholtz did not support his opinions with any empirical evidence. This problem has recently been addressed by Roederer (1975), who also basically felt that such things as as the onset envelope of a particular complex tone provides important sources of information, but again without any empirical evidence.

A recent study by Bregman and Pinker (1978) investigated the competition between "vertical" fusion of simultaneous tones into a complex tone and the tendency for a pure tone to split from its simultaneous pair and group into a separate "horizontal" stream with a pure tone that preceded the simultaneous pair. One of the important factors seemed to be the extent to which the two cooccurring pure tones started and ended together. As they became more asynchronous, there was a greater tendency for streaming to occur, and less of a tendency for 
them to fuse into a complex tone. A second important factor was whether one of the simultaneous tones was near, in frequency, to the preceding pure tone; if it was, it tended to group with the preceding tone rather than remaining as part of the complex tone. The breaking up of the complex tone by either factor caused it to sound less "rich." The present experiments were designed to further investigate the influence of various factors on the tendency of the partials of a complex tone to remain fused or to separate into parallel, cooccurring auditory streams.

In both experiments, a pure tone was alternated rapidly with a complex tone formed of three partials. A method first used by van Noorden (1975) was employed to determine whether the "target partial," the partial of the same frequency as the "captor. tone" (the preceding pure tone), was being pulled out of the complex tone to form a stream with the captor tone. This required the listener to judge the rate of onsets of the pure tone. If the pure tone appeared to occur twice as often as the complex tone, then every second occurrence of the pure tone sequence had to have been the target partial of the complex tone. Using this method, we investigated the following questions: (1) Does a partial that is asynchronous in onset or offset with others tend to "stream out" more easily? (2) Does streaming out depend on whether the higher partials are of different amplitudes than the fundamental?

\section{EXPERIMENT 1}

\section{Method}

Subjects. Fifteen subjects from the McGill University Psychology Department participated in this experiment. All subjects were paid for their services.

Apparatus. The complex tones used as stimuli in this experiment were generated by digitally synthesizing the complex waveform with a PDP-11 computer (Digital Equipment Corp.), storing the synthesized files on disk, and then outputting the files through a $\mathrm{D} / \mathrm{A}$ converter at the appropriate rate (up to $32 \mathrm{kHz}$ ). This output was then low-pass filtered at $10 \mathrm{kHz}$ to remove the noise inherent in digital synthesis, sent through an equalizer filter network to produce a flat response at the headphones (Micromonitor Electrostatic) for all frequencies used, and recorded on an Akai GX 4000.SS tape deck.

During testing, the tape output was amplified by a Sony TA-1055 stereo amplifier and presented to individual subjects through the headphones in an Audiometric testing room (No. 1202).

Stimuli. Basically, the stimuli presented on each trial consisted of a complex tone consisting of three sine tone components ( 500 , 1,000 , and $2,000 \mathrm{~Hz}$ ) alternating repeatedly with a single sine tone $(500,1,000$, or $2,000 \mathrm{~Hz})$, referred to as a "captor" tone. The choice of a complex tone consisting of the fundamental and two octaves was, to some extent, arbitrary. Some informal work conducted in our laboratory suggested that the degree of consonance or dissonance of a particular complex may affect the tendency for fusion to occur. It has long been recognized that the most consonant relationship between partials occurs with octaves (Helmholtz, 1885; Seashore, 1938); octaves were, therefore, selected as a means of holding this potential variable constant. All tones included 14-msec rise/fall times' to eliminate audible clicks. The amplitudes of each of the components of the complex tone were either all the same, decreasing for the higher harmonics (if the $500-\mathrm{Hz}$ component had an amplitude of 1 , the $1,000-\mathrm{Hz}$ component had an amplitude of .5 and the $2,000-\mathrm{Hz}$ component had an amplitude of .25), or increasing for the higher harmonics (the relative amplitudes for the $500-, 1,000-$, and $2,000-\mathrm{Hz}$ components were $.25, .5$, and 1.0 , respectively). The amplitude of the captor tone was always the same as that of the partial it alternated with.

In addition, the relative onsets of the components of the complex tone were varied. Either components started simultaneously or one of the components had an onset that was asynchronous relative to the other two. When all components had simultaneous onsets, the duration of each component was $137 \mathrm{msec}$ (including the 14-msec rise time and 14-msec fall time), followed by a $137-\mathrm{msec}$ break, then the "captor" tone (which was present for $137 \mathrm{msec}$ ), and finally another $137-\mathrm{msec}$ break. So, the total time from the onset of one complex tone until the cycle was repeated was $548 \mathrm{msec}$. When one element had an asynchronous onset, it started either $35 \mathrm{msec}$ before or $35 \mathrm{msec}$ after the other components; all elements had synchronous offsets.

Thus, there were basically four factors in this experiment: (1) which element of the complex tone was repeated (the 500-, $1,000-$, or $2,000-\mathrm{Hz}$ component); (2) the relative amplitudes of the components (all the same, decreasing, or increasing); (3) the type of asynchrony (the asynchronous element began either before or after the others); and (4) which element was asynchronous (none of them, or the $500-, 1,000-$, or $2,000-\mathrm{Hz}$ component), for a total of 72 trials. Of course, when there were no asynchronous components, the stimuli were identical for both types of asynchrony.

Each trial in the experiment consisted of the following sequence of stimuli: (1) the "captor" tone (the repeated element) was presented alone as a steady-state tone for approximately $2 \mathrm{sec}$ $(2.1 \mathrm{sec}) ;(2)$ the sequence of the complex tone alternating with the captor tone for $10 \mathrm{sec}(9.8 \mathrm{sec}) ;(3)$ two rhythmic patterns, each lasting approximately $5 \mathrm{sec}(4.9 \mathrm{sec})$ and consisting of clicks. In the first pattern (A), the clicks occurred at the same rate as the complex tone's rate of repetition (this was the slower rate). The second (faster rate) pattern (B), had clicks occurring at the same rate as every occurrence of the complex tone and the sine tone it alternated with. Each of these stimulus sequences within a trial was separated by a brief pause (approximately $1 \mathrm{sec}$ ). There was a 5-sec pause between trials for the subject's response.

Procedure. The subjects were instructed to first listen to the standard (pure) tone. They then heard the complex tone alternating with the standard tone, and were to decide at what rate they heard the captor tone repeating. If they heard the captor tone repeating at the slower rate, it would indicate that the elements of the complex tone were "fused" into a perceptual unit, preventing the target partial from being pulled out into a "horizontal," temporal stream. On the other hand, if they heard the captor tone repeating at the faster rate, it would indicate a less strong "vertical" fusion of the complex tone components, in which the target partial could be pulled out of that vertical organization to become a part of a horizontal stream; i.e., it would supply an extra pure tone "beat" in every cycle of the sequence.

Subjects indicated their responses by placing a mark along a 150-mm scale, with the extremes marked "slow" and "fast," indicating the two types of rhythm. Their mark along the scale was to indicate the ease with which they heard the standard tone repeating in one rhythm or the other, with a mark in the center meaning that either rhythm could be heard equally well. The subjects were given no instructions to attempt to "set" them to perceive any one of the rhythms.

All 72 trials were presented randomly to the subjects in one session, with a short break in the middle. The subjects then returned a second day to receive a second block of all 72 trials, presented in a different random order. 


\section{Results}

The subjects' responses were scored by measuring, for each trial, the distance of their marks on the scale in millimeters. Thus, a score of 0 indicated that the slower rhythm was perceived (i.e., fusion of the complex tone components occurred), a score of 150 indicated that the faster rhythm was perceived (i.e., horizontal streaming occurred), and a score of 75 indicated that either type of rhythm could be perceived equally well.

There are a number of important results in this experiment. First, there was a greater tendency for horizontal streams to be formed when the repeated component was $500 \mathrm{~Hz}$ rather than the two higher components; the scores for the 500-, 1,000-, and $2,000-\mathrm{Hz}$ repeated elements were $110.1,74.3$, and 78.2 , respectively $[F(2,28)=14.68, p<.001]$.

There was also a significant effect of the intensity relationships of the three components $[F(2,28)=$ 20.97, $\mathrm{p}<.001]$. The scores for decreasing, equal, and increasing component intensities were 77.5, 88.0, and 97.2, respectively, showing that the greatest overall fusion of the three sine-tone components occurred when the relative amplitudes of the higher components decreased.

There was a significant interaction between the intensity variable and which element was repeated $[F(4,56)=12.43, p<.001]$; this can be seen in Figure 1. This interaction suggests that the more

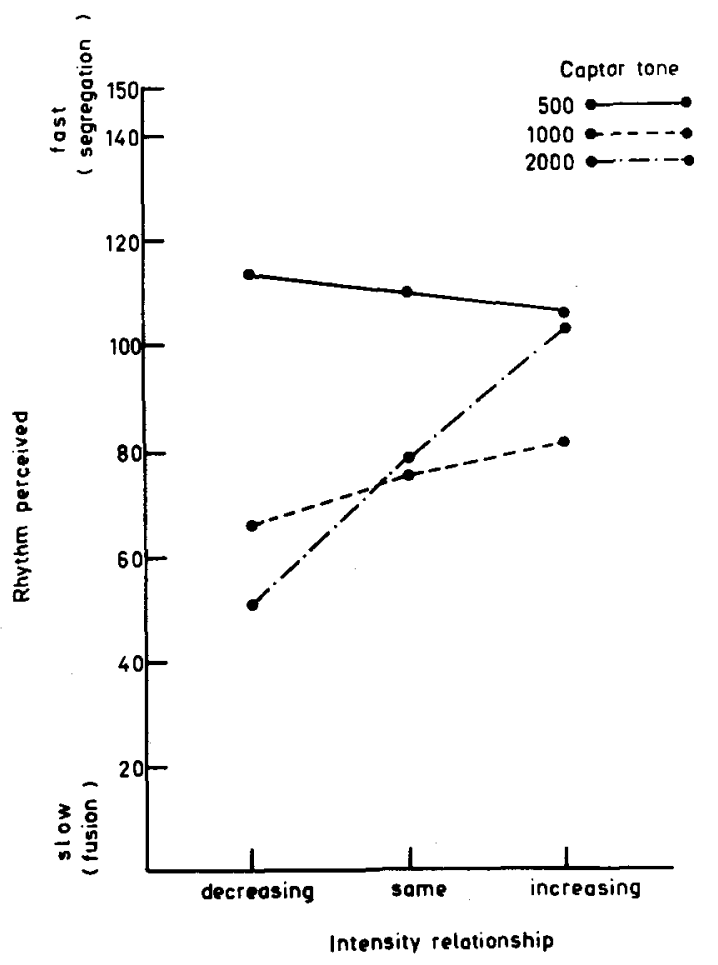

Figure 1. Interaction between the intensity relationship and which component of the complex tone was the repeated one for Experiment 1.

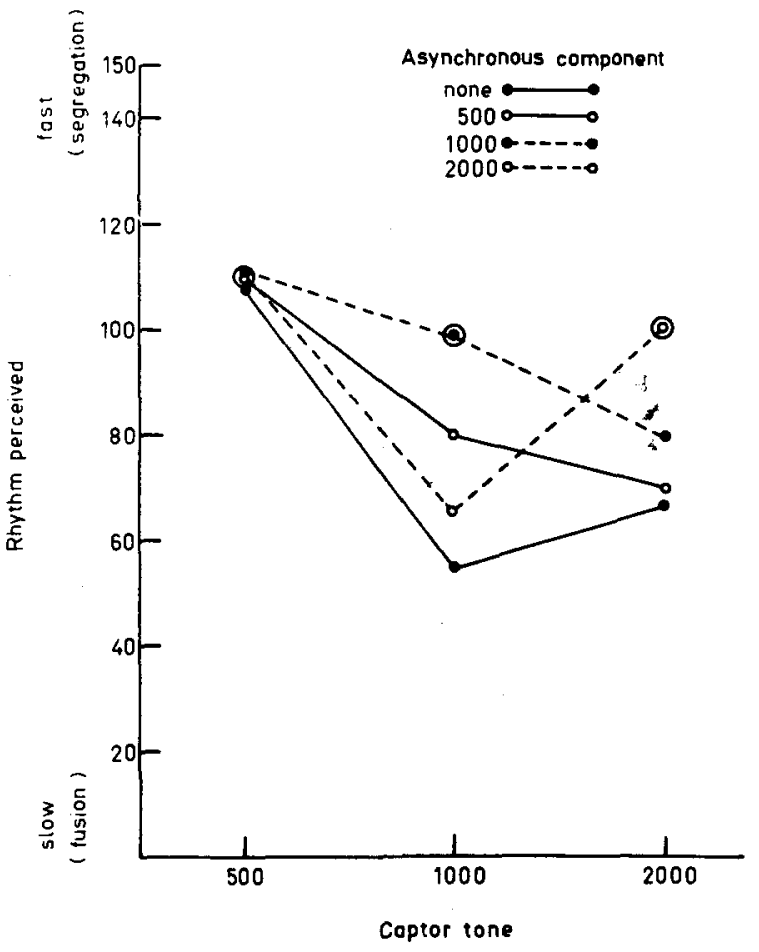

Figure 2. The interaction between which element was the repeated one and which was asynchronous, showing that, except for the $500-H z$ component, the repeated element was "pulled" into a horizontal stream much better when it was asynchronous (the circled points).

intense a partial, the easier it is pulled into a stream, with higher partials generally being more difficult to pull into a stream than the fundamental.

There was a significant main effect for which component was asynchronous $[F(3,42)=12.44, p<.001]$; the greatest tendency for horizontal streams to be formed occurred when the $1,000-\mathrm{Hz}$ component was asynchronous (96.2), followed by the $2,000-\mathrm{Hz}$ component (91.8), the $500-\mathrm{Hz}$ component (86.1), and finally no asynchronous components (76.0). This seems, however, to be an incidental result of the interaction between which component was asynchronous and which one was repeated, as shown in Figure 2; this interaction was highly significant $[F(6,84)=9.92, p<.001]$. As can be seen in Figure 2, there was a greater tendency for a horizontal stream to be formed when the repeated component was asynchronous (circled points in the figure) than when it was synchronous. However, there was also a significant three-way interaction between these factors and the type of asynchrony $[F(6,84)=3.36, p<.01]$, as shown in Figure 3. This figure shows that the effect of asynchrony in pulling out the repeated component into a horizontal stream (circled points in the figure) is stronger when the asynchronous component starts before the other components of the complex tone rather than after them. There was no overall main effect of the type of asynchrony. 


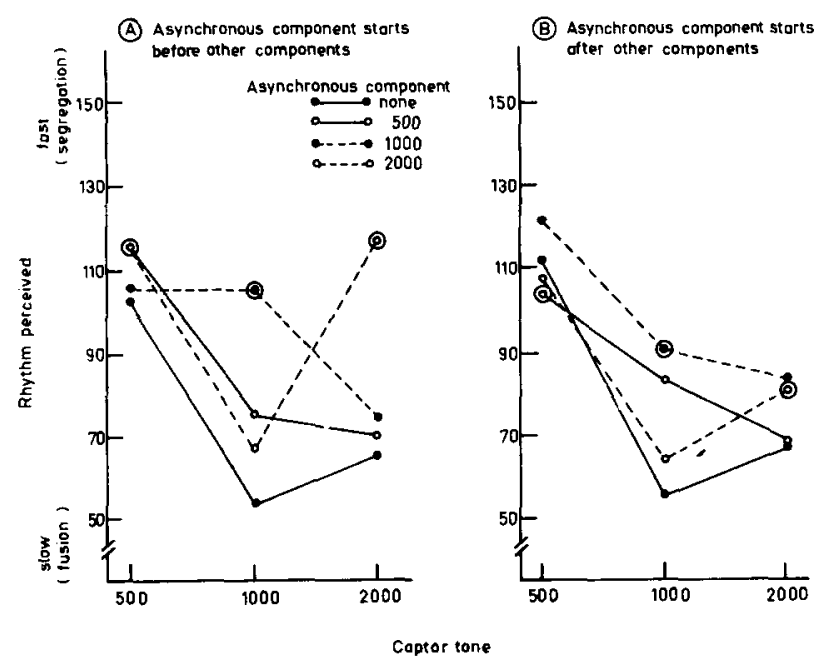

Figure 3. Three-way interaction showing that the asynchrony effect was stronger when the asynchronous element started before the others rather than after (circled points are repeated, asynchronous components).

Finally, there was a significant three-way interaction between the frequency of the repeated component, which component was asynchronous, and the relative intensities of the components $[F(12,168)=2.63$, $p<.01]$; this interaction is shown in Figure 4. This figure shows that the basic interaction between asynchrony and which element of the complex is repeated (shown in Figure 2) is affected by the intensity relationship of the components. As the intensity relationship changes from a decreasing one to an increasing one, it becomes increasingly easier to pull out the asynchronous element into a horizontal stream.

\section{Discussion}

There are a number of important results in this experiment. First of all, finding that the fundamental can easily be pulled into a "horizontal" auditory stream is probably not terribly surprising; Plomp (1964) found that it was easiest for subjects to "hear out" the fundamental and lowest partials of a complex tone, and that this became increasingly difficult for the higher partials. Of somewhat greater interest is the role that the relative amplitudes of the different sine-tone components seem to play. The greatest amount of fusion of the complex tone occurred when the amplitude decreased for the higher components. This, of course, is more or less the state of affairs for naturally produced complex sounds, although one often finds that a higher partial may be more intense than a lower one (Fletcher, 1952; Winckel, 1967); it may be that the auditory mechanism has evolved to fuse tones of this type. Producing an unusual type of amplitude relationship, such as increasing amplitude for the higher components, decreases the fusion of the complex, and also greatly increases the ability of the repeated tone to pull out the $2,000-\mathrm{Hz}$ component, normally much more difficult to pull into a horizontal stream.

The other results of interest concern the effects of asynchrony on streaming vs. fusion. An element that had an asynchronous onset relative to the other components was always easier to pull into a horizontal stream than when it started synchronously (except for the $500-\mathrm{Hz}$ tone, which was always fairly easily pulled into a horizontal stream). Secondly, this effect was greater when the onset of the asynchronous partial was before the others rather than after. Since offsets were always synchronous, the type of asynchrony is confounded with the duration of the component, with those components having late onsets also being shorter than those with earlier onsets, resulting in a lower total energy for these components. Although it is unlikely that this had any significant effect, since the difference in energy between the shortest component and the longest component would only result in an average change of $1.3 \mathrm{~dB}$, it is possible that this could reduce the detectability of the shorter component. This might decrease its tendency to be pulled into a horizontal, temporal stream.

\section{EXPERIMENT 2}

It seems clear, from studies such as Experiment 1 and that by Bregman and Pinker (1978), that a component of a complex tone which is asynchronous relative to the other components is more easily pulled into a horizontal stream. One variable which has not been investigated systematically is any possible difference between onset and offset asynchronies. In

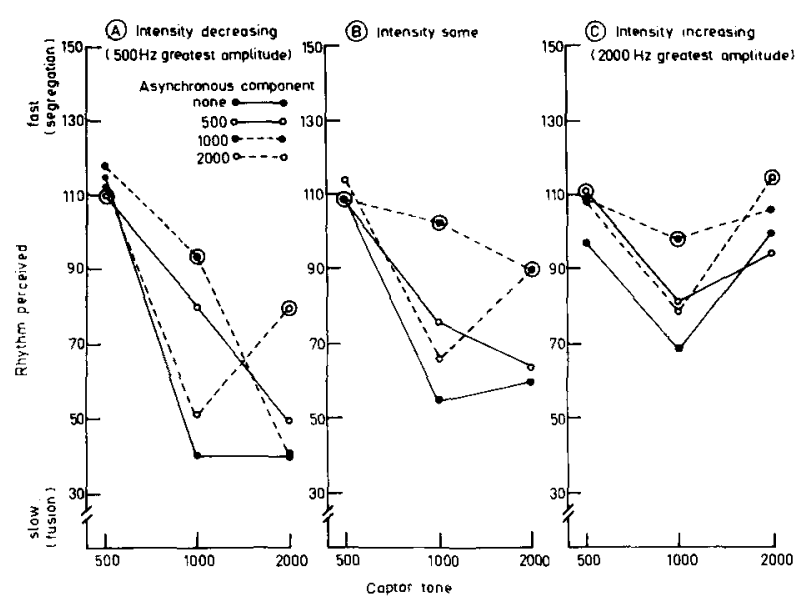

Figure 4. The interaction between the frequency of the repeated element, asynchronous element, and intensity relationship for Experiment 1. 
Bregman and Pinker's stimuli, all asynchronies were due to both onset and offset asynchronies; in Experiment 1, only onset asynchronies were investigated. Experiment 2 was designed to investigate in detail the effect of synchronous vs. asynchronous onsets and offsets on the competition between fusion and streaming of components of a complex tone.

\section{Method}

Subjects. Fifteen subjects, recruited from the campus of St. Francis Xavier University during a summer session, participated in this experiment. All subjects were paid for their services.

Apparatus. The apparatus used to generate and record the stimuli used in this experiment was identical to that used in Experiment 1. The stimuli were played to subjects, seated in a small room, on a Sony TC-540 stereo tape recorder through Sharpe MKII stereo headphones. All stimuli were played through a Southwest Technical Products 216/A stereo equalizer filter, set to produce equal amplitudes of all three sine-tone components at the headphones (as measured by a General Radio Type 1551-C sound level meter through a 6-cc coupler).

Stimuli. The stimuli used in the present experiment were basically similar to those used in Experiment 1, consisting of a complex tone (with $500-, 1,000-$, and $2,000-\mathrm{Hz}$ components) alternating with a single sine tone $(500-, 1,000-$, or $2,000-\mathrm{Hz})$. Rise/fall times and basic tonal durations were the same as those used in Experiment 1 . In the present experiment, however, the asynchronous component was always the frequency of the repeated element.

The variable of particular interest in this experiment was that of asynchrony. The stimuli can be described in terms of three additional variables. First, the stimuli varied according to the type of asynchrony. Lead asynchrony refers to the asynchronous element either starting before the other elements (onset lead) or ending before the other elements (offset lead). Lag asynchrony designates the situation in which the asynchronous element either starts after the others (onset lag) or ends after the others (offset lag). Secondly, there were three levels of onset asynchrony $(0,35$, and $69 \mathrm{msec})$, and finally, three levels of offset asynchrony $(0,35$, and $69 \mathrm{msec}$ ). These three variables were combined factorially to produce 18 different stimulus types; each of these can be seen in Figure 5 (note that this figure illustrates the different types of asynchrony for the $1,000-\mathrm{Hz}$ component). Since the asynchronous component could be any of the three partials, there were 54 different trials, presented to subjects in three blocks, in a different random order for each block, for a total of 162 trials. Half of these trials were presented to subjects one day and the other half the following day.

Procedure. The procedures used in this experiment were slightly different from those used in Experiment 1. Unlike Experiment 1, almost all of the stimuli in the present experiment have a repeated component with asynchronous onsets, or offsets, or both. Preliminary investigation revealed that this resulted in a subject's almost always perceiving the faster rhythm (using the methodology of Experiment 1), thus making it difficult to see any differences between the conditions of the present experiment. As a result, the procedures were changed for this experiment. In each trial, the subject first heard only the slow rhythm of clicks; this was followed by the standard tone, and then the complex tone alternating with the standard (captor) tone. The subjects were asked to concentrate on the captor tone when alternating with the complex tone and to try to make it sound like the slow rate of the clicks (i.e., try to hear the repeated component as fused with the other two components and not hear it segregate into a separate, horizontal stream). In a number of informal investigations we have made in our laboratory, we have noticed that the tendency for auditory streams to form is under a certain degree of "voluntary" control on the part of the listener; this has also been noted by van Noorden

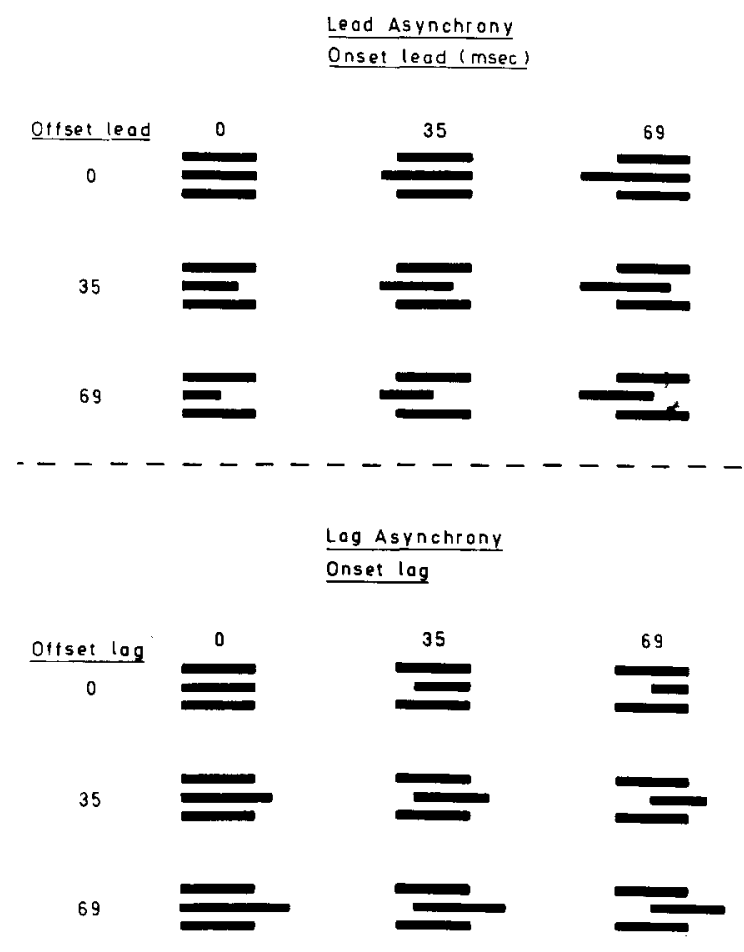

Figure 5. Schematic diagrams of stimuli used in Experiment 2.

(1975). Thus, even though the overall tendency is for subjects to hear most of these stimuli as streaming, a method such as this allows us to identify the relative ease with which fusion vs. streaming occurs.

The subjects indicated their responses by placing a mark along a horizontal scale which ranged from 1 to 14 , with 1 indicating that it was very easy to hear the captor tone repeating at the rhythm of the clicks (i.e., the subject could make all three components fuse and prevent streaming), while 14 indicated that it was very difficult to hear that rhythm (i.e., that the tendency for the component to be "pulled out" into a stream was too strong to be overcome by any voluntary control on the part of the subject).

\section{Results}

An analysis of variance revealed, first of all, that there was a significant main effect of the duration of onset asynchrony, with a greater tendency for streaming to occur as the duration of onset lead time increased $[\mathrm{F}(2,28)=10.08, \mathrm{p}<.001]$. In addition, there was a significant main effect of the duration of offset asynchrony $[F(2,28)=4.06, p<.05]$; offset asynchrony caused a greater tendency for streaming to occur, compared with stimuli having 0 -msec offset asynchronies. Both of these main effects can be seen in Figure 6. In addition, the analysis revealed interaction between both of these variables and the type of asynchrony (lead or lag); these interactions are also apparent in Figure 6. First, onset asynchrony duration basically had its effect only with stimuli having lead asynchrony $[\mathrm{F}(2,28)=6.54, \mathrm{p}<$ 


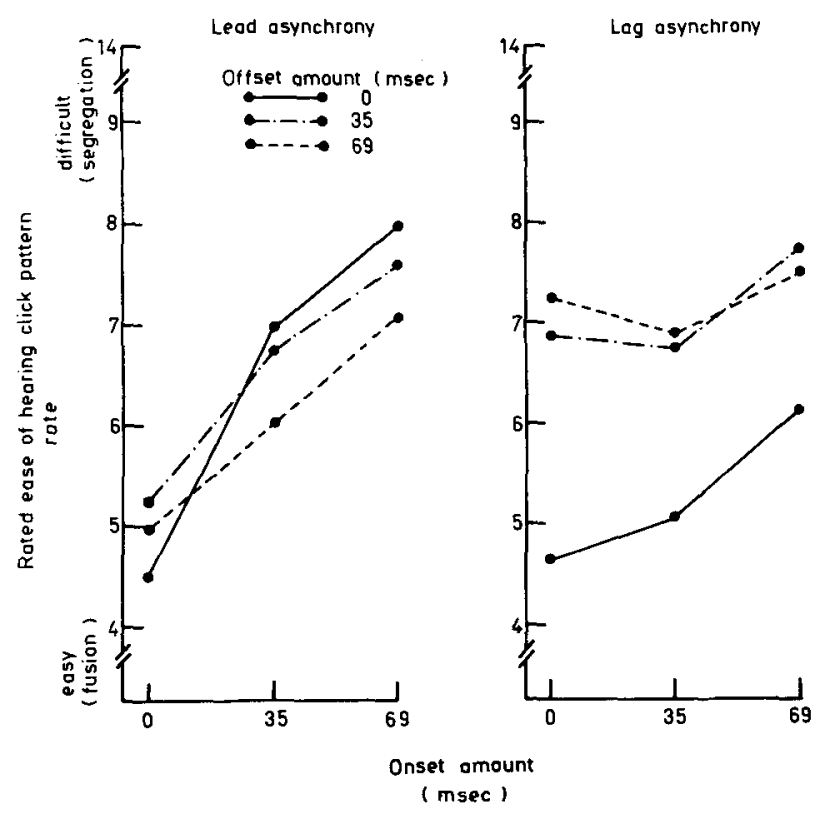

Figure 6. The three-way interaction between type of asynchrony, amount of onset asynchrony, and amount of offset asynchrony for Experiment 2.

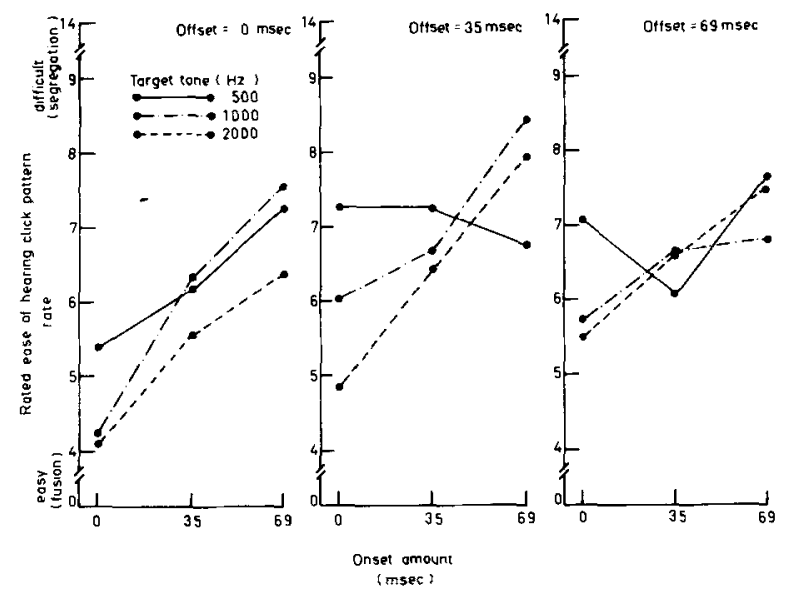

Figure 7. The three-way interaction between amount of onset asynchrony, amount of offset asynchrony, and target tone frequency for Experiment 2.

.0051. On the other hand, of fset asynchrony duration had an effect only with stimuli having lag asynchrony $[\mathrm{F}(2,28)=6.64, \mathrm{p}<.005]$.

In addition to these effects, there were a number of interactions between these variables and which component of the complex was the target tone. First, as can be seen in Figure 7, there was a general tendency for the duration of onset asynchrony to have an effect on the two upper partials, but little, if any, effect on a $500-\mathrm{Hz}$ target tone $[\mathrm{F}(4,56)=2.67$, $p<.05]$. These two variables also interacted with the duration of offset asynchrony $[F(8,112)=2.37$, $\mathrm{p}<.05$ ] (again, see Figure 7). The figure reveals that, in general, the effect of increasing the duration of offset asynchrony caused a decrease in the effect of the duration of onset asynchrony.

There was a significant three-way interaction between the duration of onset asynchrony, the type of asynchrony, and the target tone $[F(4,56)=2.84$, $\mathrm{p}<.05$ ] shown in Figure 8. This figure shows that the interaction between onset asynchrony duration and type of asynchrony occurs for the 1,000- and $2,000-\mathrm{Hz}$ target tones, but not for the fundamental, $500-\mathrm{Hz}$ tone.

Finally, there was a significant three-way interaction between the duration of offset asynchrony, the type of asynchrony, and the target tone $[\mathrm{F}(4,56)=$ $3.27, \mathrm{p}<.025]$ as presented in Figure 9. This figure is quite similar to Figure 8 , in that it shows that the

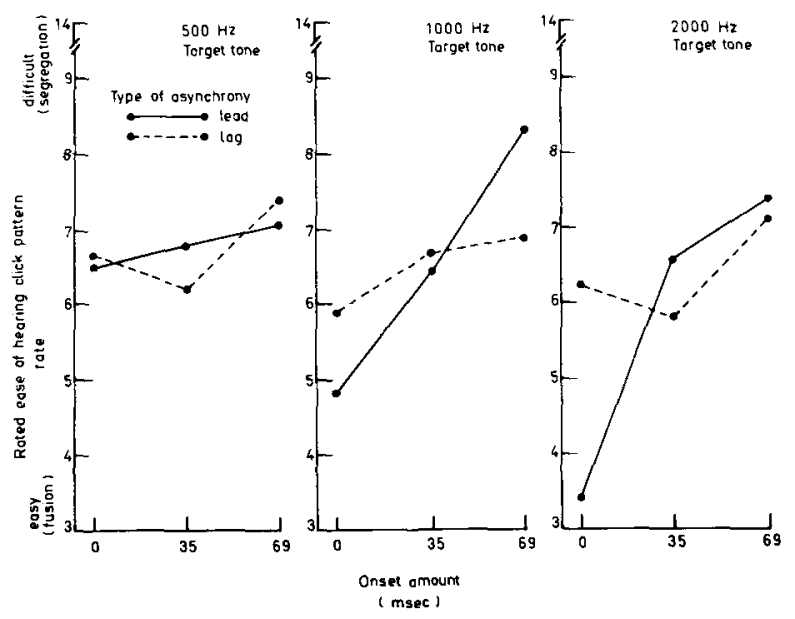

Figure 8. Three-way interaction between amount of onset asynchrony, type of asynchrony, and target tone frequency for Experiment 2.

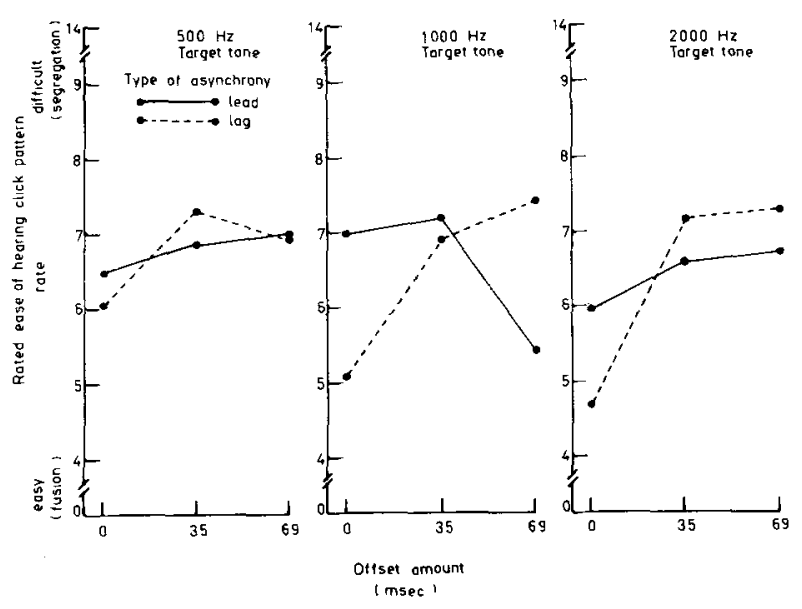

Figure 9. Three-way interaction between amount of of fset asynchrony, type of asynchrony, and target tone frequency for Experiment 2. 
interaction between offset asynchrony duration and the type of asynchrony occurred for the 1,000- and $2,000-\mathrm{Hz}$ target tones, but not for the fundamental.

\section{Discussion}

There are several results of interest in this experiment. First, an important result is that, for asynchrony to be effective in pulling the target tone into a horizontal stream, the target tone must either start before the other components or end after them; i.e., onset asynchrony duration had an effect only with lead asynchrony, and offset asynchrony duration had an effect only with lag asynchrony. This, of course, is similar to the results obtained in Experiment 1 with onset asynchronies. A possible explanation for this effect is that when the onset of the target tone occurs after the onset of the other components or when the offset occurs before the offsets of the other components, the other components mask the onset (or offset) of the target tone, thus reducing the effectiveness of the asynchrony. Also, it is possible that an "auditory continuity" effect (Bregman \& Dannenbring, 1977; Dannenbring, 1976) is occurring here, and that the asynchronous component is perceptually longer than its physical duration would indicate. Again, this would reduce any potential effect of asynchrony. At this point, such a notion is simply speculation and would have to be tested by further research.

Another factor which may be affecting some of the results just described is the actual duration of the target tone. For example, Figure 6 shows that for stimuli with 0 msec offset asynchrony duration, there was a greater tendency overall for segregation to occur for lead asynchronous stimuli than for lag asynchronous stimuli. As can be seen in Figure 5, these stimuli under lag conditions are shorter in duration than the 0 -msec offset stimuli under lead conditions. It may be that the shorter component, having less total energy, is somewhat less detectable, and thus more easily fused with the other components, a point which was made in discussing the results of Experiment 1 . One way to test this hypothesis would be to look directly to the effect of the duration of the asynchronous component, something that was not a part of the original analysis of variance. In the present experiment, there are five different durations of the asynchronous component: $69,103,137$, 171 , and $205 \mathrm{msec}$. A one-way analysis of variance was conducted in which all of the stimuli were placed into these five categories according to the duration of the target element. This analysis revealed a significant difference between the mean scores $[F(4,265)$ $=2.41, \mathrm{p}<.05]$, which can be seen in Figure 10 . This figure suggests that there is an increased tendency for streaming to occur as the duration of the asynchronous component increases, which agrees with the notion that the longer component, being more salient and detectable, should be more easily pulled into a horizontal stream (or, conversely, more difficult to fuse with the other components).

Finally, the general conclusion to be drawn from the various interactions that occurred with the frequency of the target tone is that the $500-\mathrm{Hz}$ tone was not as affected by the other variables, a result that again agrees with the results of Experiment 1.

\section{GENERAL DISCUSSION}

In this paper, we have tried to look at a number of variables which might potentially affect the tradeoff between streaming and fusion in a temporal pattern of complex waveforms. It is probably useful at this point to try to summarize some of the major results of this research and, further, to look at the implications of this research for the perception of real-world auditory events.

Probably the most important finding of this research is that asynchronous components are, in general, more easily pulled into horizontal streams than synchronous components, which supports the earlier speculations by Helmholtz (1885) and Roederer (1975) and the investigations by Bregman and Pinker (1978). The present research, however, is important in demonstrating effects of such things as the amount of asynchrony, whether the asynchrony is due to

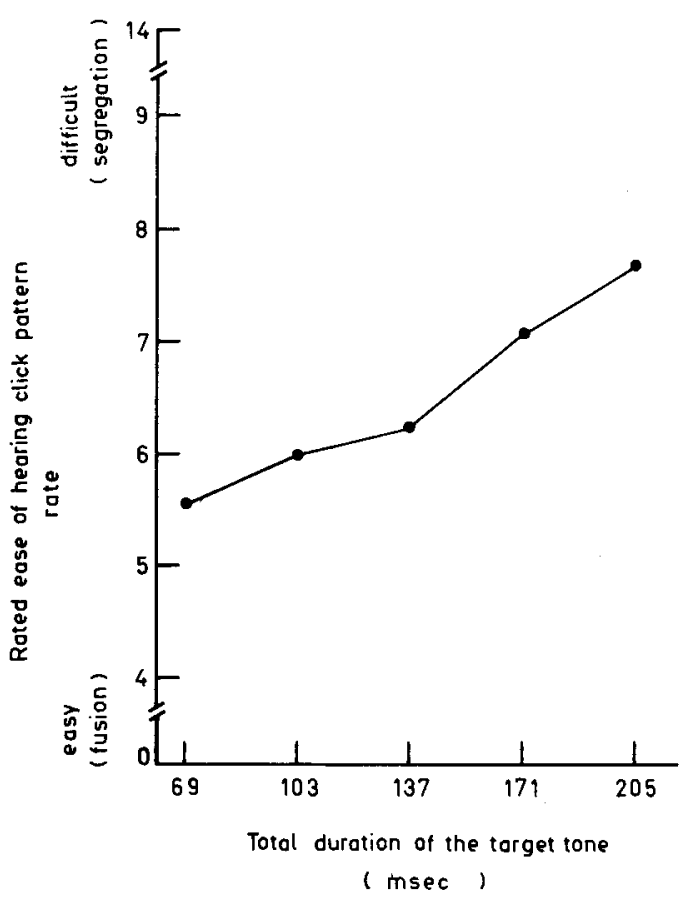

Figure 10. Effect of the duration of the target tone on fusion vs. streaming in Experiment 2. 
tonal extensions or to shorter tonal components, onset vs. offset asynchronies, and such things as the intensity relationships of the partials of the complex tone.

In addition, this research demonstrates that the use of perceived rhythm as a dependent variable seems to be a fairly useful technique. However, one should keep in mind that real-world auditory events do not consist of such highly predictable patterns as the alternation of a complex tone with one of its partials. Indeed, the predictability of such a pattern may result in certain variables affecting streaming or fusion which may not be important in a more natural environment. For example, one of the interesting results of this research was that offset asynchrony, especially when the offset occurs after the offsets of the other components, results in as strong a tendency for streaming to occur as under conditions of onset asynchrony. This seems somewhat surprising, since it suggests that the auditory system decides at the point of tonal offset that one of the partials that had already been input should, in fact, belong to a different horizontal stream and not to the complex. One might think that such a delay in assigning the partial to a particular perceptual group should be somewhat more difficult than the more immediate, sequential process that should be possible with onset asynchronies, and should thus result in less of an effect for offset asynchronies; this, of course, did not occur. However, due to the artificial, cyclical nature of the stimuli, it is possible that a potentially smaller effect of offset asynchrony "builds up" over the course of the 10-sec presentation of the complex-captor sequence, so that by the end of the $10 \mathrm{sec}$ there is no difference between onset and offset asynchronies. The answers to such questions, of course, require additional research.

\section{REFERENCES}

Bregman, A. S. The formation of auditory streams. In J. Requin (Ed.), Attention and performance VII. Hillsdale: Erlbaum, 1977.

Bregman, A. S., \& Campbell, J. Primary auditory stream segregation and perception of order in rapid sequences of tones. Journal of Experimental Psychology, 1971, 89, 244-249.

Bregman, A. S., \& Dannenbring, G. L. The effect of continuity on auditory stream segregation. Perception \& Psychophysics. 1973, 13, 308-312.

Bregman, A. S., \& Dannenbring, G. L. Auditory continuity and amplitude edges. Canadian Journal of Psychology, 1977, 31, 151-159.

Bregman, A. S., \& Dannenbring, G. L. Auditory streaming. Scientific American, in press.

Bregman, A. S., \& Pinker, S. Auditory streaming and the building of timbre. Canadian Joumal of Psychology, 1978, 32. 19-31.

Bregman, A. S., \& Rudnicky, A. I. Auditory segregation: Stream or streams? Journal of Experimental Psychology: Human Perception and Performance, 1975, 1, 263-267.

Dannenbring, G. L. Perceived auditory continuity with alternately rising and falling frequency transitions. Canadian Journal of Psychology, 1976, 30, 99-114.

Dannenbring, G. L., \& Bregman, A. S. Effect of silence between tones on auditory stream segregation. Journal of the Acoustical Society of America, 1976, 59, 987-989. (a)

Dannenbring, G. L., \& Bregman, A. S. Stream segregation and the illusion of overlap. Joumal of Experimental Psychology: Human Perception and Performance, 1976, 2, 544-555. (b)

Fletcher, H. Speech and hearing. New York: van Nostrand, 1952.

Helmholtz, H. On the sensations of tone (2nd English ed., Trans. by A. J. Ellis, 1885; reprinted by Dover Publications, 1954).

NoORden, L. P. A. S. van. Temporal coherence in the perception of tone sequences. Published PhD thesis, Institute for Perception Research, Eindhoven, Holland, 1975.

Plomp, R. The ear as a frequency analyzer. Journal of the Acoustical Society of America, 1964, 36, 1628-1636.

ROEDERER, J. G. Introduction to the physics and psychophysics of music. New York: Springer-Verlag, 1975.

SEashore, C. E. Psychology of music. New York: McGraw-Hill, 1938.

WincKel, F. Music, sound and sensation: A modern exposition. New York: Dover Publications, 1967.

\section{NOTE}

1. The rather unusual stimulus durations used in these experiments are due to the operation of the synthesis program. In outputting the synthesized stimuli, it was necessary for the computer to output a complete, fixed-size buffer. To avoid problems (i.e., blank spaces) that would result if a synthesized stimulus did not completely fill a buffer, the program could automatically "stretch" the durations of the stimuli (i.e., add more cycles to a particular tone) until it exactly filled the buffer. In the present experiments, all stimuli were "stretched" by a factor of 1.365 , so that, for example, a tone specified to the computer as $100 \mathrm{msec}$ would, in fact, have a duration of $137 \mathrm{msec}$ (all fractions are rounded up). Due to rounding and the fixed-sized output buffer, the fall time of the captor tone was reduced to $10 \mathrm{msec}$. This did not, however, result in a perceptible click on tonal offset.

(Received for publication March 7, 1978; revision accepted August 1, 1978.) 